\title{
The Multimedia Home Platform (MHP) Framework for Web Access through Digital TV
}

\author{
Alberto Gil, José J. Pazos, Jorge García, Rebeca P. Díaz, Ana Fernández, and \\ Manuel Ramos
}

ETSE Telecomunicación, University of Vigo, Vigo, Spain

agil@det.uvigo.es

\begin{abstract}
The DVB Consortium has recently published the MHP standard to regulate the execution of interactive applications in digital $\mathrm{TV}$, including an Internet profile for web access. In this communication, that profile is analysed.
\end{abstract}

\section{Introduction}

Nowadays, digital technology is greatly enhancing traditional broadcasting: more channels in the same bandwidth, better image quality, hi-fi sound, etc. In the near future, watching television will be enhanced with a lot of amazing possibilities: complementary information to audiovisual contents, electronic program guides, selection of properties in configurable contents (language, camera angle or particularised advertisement), games, etc. These services are implemented by means of multimedia procedural applications being received in the digital transport stream. To guarantee competition (in order to reduce costs and prices), it is necessary to achieve compatibility in the computing platform that applications will find when they arrive at the digital TV set. The MHP standard [1] has been developed to standardize all necessary elements to promote compatible equipment and contents: the code of the MHP applications (Java), the execution context, the APIs supported in the SetTop box (STB), the life cycle of the applications, the signalling of the operator, etc.

But this standardization is not enough to achieve a successful integration of this new technology in the TV market. Content providers demand the introduction of new value-added services which users are willing to pay for, as Internet is. Besides, DVB considers it is necessary to equip digital TV with a "super teletext" system for applications or content providers to present structured stylised information to users.

So, MHP pays special attention to the specification of all necessary details to integrate in its computing model a mechanism to access to Internet or to browser over information structured in a similar way, adopting W3C standards as a starting point.

\section{Internet Access through TV: The MHP Approach}

We will concentrate on three different aspects of WWW browsing technologies:

- DVB has chosen the W3C XHTML Modularization standard to define DVB-HTML, the markup language that an MHP Internet browser must understand. It is composed of a set of modules that take into account the critical differences between computers 
- and TV sets (computing power, screen resolution, viewing distance, etc.). A DVBHTML application consists of a set of DVB-HTML documents forming a directed graph, and permits the service provider to send formatted and stylised data (several related DVB-HTML documents) in a packed and standard format. Even more important, the downloading, decoding, rendering and interaction processes of DVBHTML applications must follow a well-defined life cycle in the user agent, which can be commanded from the content provider or by any other MHP application.

- In order to get a stylised rendering of a DVB-HTML document, the W3C CSS2 standard has been adopted. All the selectors defined in CSS2 must be included and a new media device is defined, $d v b$ - $t v$, to identify tv-like devices: low resolution and computing power, limited scrollability, reduced interfaces, etc. Opacity is specifically addressed (and composition rules defined) as blending contents with background is a very frequent graphic operation in TV. A new at-rule is introduced (@viewport) where new pseudo-classes are defined to adapt to some parameters tvrelated: device aspect $(4: 3,16: 9 \ldots)$, resolution, etc. Regarding fonts, only support for Tiresias type, plain, with four large sizes $(24,26,31$ and 36pt) is mandatory.

- MHP has adopted the W3C DOM and the ECMA ECMAScript standards to offer programmatic access to the internal logical structure derived from DVB-HTML documents, CSS2 decoration rules included. The fundamental structure has been extracted from the W3C DOM Level 2. The adopted events module defines mouse events as optional and includes new system events for the user agent to communicate life cycle transitions to the DVB-HTML application. A new kind of event (triggers) is defined for the user agent to deliver synchronization signals (in DOM events form) received from the service operator. All the interfaces must be offered as ECMAScript and Java interfaces, as well as an interface for scripts to access to all MHP APIs, which permits the implementation of hybrid applications that can divide their functionality between a declarative part (DVB-HTML containing scripts) and a procedural part (the Java code of MHP applications).

\section{Conclusions}

The MHP standard is the first attempt to standardize the computing platform of a STB with Internet access. The standard, quite ambitious, seems to be suitable for rendering documents specifically designed for this media. But Internet contents are more complex and heterogeneous, which may imply delays and differences in appearance of results in TV. The different evolving rate and life-span of TVs and computers will power technologies to particularize answers according to the client capabilities.

\section{References}

1. DVB Consortium: Multimedia Home Platform 1.1. http://www.mhp.org, (2001) 